\title{
Calculation and Analysis of Aircraft Pollutant Emission Based on Time Wake Separation Mode under Coastal Airport and Headwind Conditions
}

\author{
Pan Wei-Jun $\mathbb{D}^{1},{ }^{1}$ Zhang Heng-Heng $\mathbb{D}^{1},{ }^{1}$ Zhang Xiao-Lei $\mathbb{D}^{1,2}$ and Wu Tian-Yi ${ }^{1}{ }^{1}$ \\ ${ }^{1}$ College of Air Traffic Management, Civil Aviation Flight University of China, Guanghan 618307, China \\ ${ }^{2}$ Department of Radiology, Second Affiliated Hospital of Shantou University Medical College, Shantou 515041, China \\ Correspondence should be addressed to Zhang Heng-Heng; gangshikonglaol@163.com and Zhang Xiao-Lei; \\ bmezhang@vip.163.com
}

Received 17 May 2021; Revised 1 July 2021; Accepted 17 August 2021; Published 25 August 2021

Academic Editor: Xianhua Wu

Copyright (C) 2021 Pan Wei-Jun et al. This is an open access article distributed under the Creative Commons Attribution License, which permits unrestricted use, distribution, and reproduction in any medium, provided the original work is properly cited.

During the final approach, the headwind leads to a reduction of landing rate, which affects the achieved capacity and the predictability of operation, time, fuel efficiency, and environmental pollution. Under headwind conditions, ground speed decrease results in increased flight time. Time-based separation (TBS) changes the separation rule of the final approach, which changes the distance separation between two aircrafts into a time separation. This paper introduces the time-based separation (TBS) based on the distance-based separation (DBS). According to the aircraft landing schedule of each airport, the ICAO (International Civil Aviation Organization) aircraft engine emission database, Boeing Fuel Flow Method 2 (BFFM2), and meteorological data of Pudong airport, this study uses the modified P3-T3 aviation pollutant emission model to calculate, respectively, the fuel consumption and pollutant emissions based on distance separation mode and time separation mode. According to the calculation results, TBS operation mode can save $32.52 \%, 19.12 \%$, and $30.41 \%$ fuel, reduce $28.93 \%, 17.9 \%$, and $29.29 \%$ CO, $31.02 \%, 19.36 \%$, and $33.78 \%$ HC, $30.85 \%, 16.42 \%$, and $28.67 \%$ NOx, respectively, compared with the DBS operation mode at three stages of the day. It ends that TBS has an obvious optimization effect on fuel consumption and pollutant emission compared with DBS from data.

\section{Introduction}

The aviation pollutants mainly include nitrogen oxides (NOx), carbon monoxide (CO), and unburned hydrocarbons (UHC) [1]. The International Civil Aviation Organization (ICAO) stipulated that the engine performance and fuel consumption rate need to be improved continuously in the industry, and aircraft should reduce the emission of pollutants to meet the increasingly stringent airworthiness requirements [2].

When the headwind speed increases, the reduction of the ground speed of the aircraft leads to an increase in flight time and a decrease in the arrival rate, which not only affects the current stable airport capacity but also affects the predictability of operation, time, fuel efficiency, and environmental pollution [3]. The TBS had been evolved into a concept by the National Air Traffic Service (NATS) and Leidos (formerly Lockheed-Martin) as a separate system to fully unlock the runway capacity irrespective of wind conditions [4]. In the frame of SESAR Time-Based Separation (TBS) concept was used to provide a consistent time spacing between aircraft in airports to increase runway throughput according to Morris [5]. The Time-Based Separation concept was proposed as a logical way to solve possible conflicts between an RPAS and a civil aircraft by assigning a controlled time to overfly (CTO) to RPAS. In 2015, the system was operational at London's Heathrow Airport after over 150000 assessments on wake vortices of inbound flights. This system used time separation instead of distance separation to increase runway usage and ensure safety [6]. The Tribhuvan International Airport (TIA) used the time-based separation for two consecutive aircraft in approach. Nishan published an 
article in 2020 and concluded: An average daily time lag of 79 minutes is observed between DBS and TBS. Hence, implementation of the ETBS system can save TIA almost 20 days of operation annually [7].

The ICAO stipulated the emission standards of $\mathrm{CO}$, UHC, and NOx in the takeoff, climb, approach, and ground taxiing stages of the standard LTO (landing and takeoff) cycle. Xia estimated the pollutant emissions during the takeoff and landing cycle of Chinese civil aviation airports according to the engine emission data published by ICAO, which provides the emission data of different pollutants for this paper [8]. Huang studied the NOx emission distribution over China by using the fuel flow method, which provides a reference for the establishment of the fuel flow method in this paper [9]. According to the actual flight parameters, Wei estimated the pollutant emissions of each flight stage by using the fuel flow method, which provides the engine thrust setting and flight time of each flight stage for this paper [10]. According to the schedule, route setting of CAAC, and the characteristics of aircraft and engine, Huang evaluated the NOx stock of flight emissions in 2001 by using the emission database published by ICAO and NASA, which provides a reference for the final calculation of the total pollutant emission in each stage of for this paper [11]. However, there were few studies on the direct estimation method and P3-T3 method based on pollutant generation mechanism in China.

There are a direct method and relative method in the calculation of aviation pollutants. The direct method needs to be based on combustion chemical reaction dynamics. Since the calculation process involves the data of combustion chamber design parameters and fuel atomization characteristics, this method is difficult to model and calculate. The model is usually only applicable to specific working conditions, so the calculation error is significant under other working conditions [12]. In this study, the relative method uses the known emission indexes of the engine under various thrust conditions on the ground, then modifies the model according to the ratio of some key thermal parameters of the aircraft under high altitude and ground conditions, and finally calculates the emission indexes of the starting engine under various actual conditions. These key parameters mainly include the total temperature (T3) and total pressure (P3) at the inlet of the combustion chamber. The above key parameters are easy to obtain. Therefore, this method is also called the P3-T3 method [13]. According to the P3-T3 emission index and Boeing Fuel Flow Method 2 (BFFM2) calculation model, this study calculates the reduced operation time based on TBS mode compared with the DBS mode under the same landing sorties and obtains the pollutant emissions under the two modes. It is further concluded that the TBS operation mode has an obvious effect on reducing pollutant emission compared with the DBS operation mode.

This paper theoretically studies the saved time by the TBS operation mode compared with the traditional DBS operation mode. Under the condition of the same number of approaching flights, the saved time by the TBS operation mode compared with the DBS operation mode is converted into fuel consumption reduction and pollutant emission.
Finally, the paper evaluates the TBS mode's significant energy-saving and emission reduction effect compared with the DBS mode. The TBS operation mode will significantly contribute to the energy conservation and emission reduction of the airport. The TBS mode can reduce fuel consumption by at least $30 \%$ and pollutant emissions by $25 \%$. The application of this method can reduce aircraft flight time, improve operational efficiency, reduce fuel consumption, and reduce pollutant emission for coastal airports under headwind conditions. The structure chart of the article is shown in Figure 1.

\section{Materials and Methods}

\subsection{Estimation of Pollutant Emission Index of Aeroengine}

2.1.1. Model Assumptions. A. All flights operate in the international standard atmosphere, and the changes of humidity and temperature are ignored in a day. B. In this study, the standard LTO cycle specified by ICAO is adopted, and the defined emission is $915 \mathrm{~m}$ (3000 feet) from the surface to the top of the atmospheric boundary layer regardless of the aircraft engine emission under cruise conditions. The approach, taxi, takeoff, and climb times are taken from the standard LTO cycle specified by ICAO. The approach process requires $30 \%$ of the engine thrust and lasts for 4 minutes [10]. C. The flight speed and engine fuel flow remain unchanged during the approach. D. The average approach speeds of different aircraft types are shown in Table 1. E. The chemical reaction between pollutants and atmospheres is ignored.

\subsubsection{Calculation of Fuel Consumption and Pollutant} Emission of Aeroengine. The LTO can describe all the aircraft activities cycle at the airport [14]. This study uses the specified standard cycle, which ranges from the surface to the top of the atmospheric boundary layer (3000 feet). The operational status, thrust, and operation time of each stage are shown in Table 2. The fuel consumption and emission index of the aircraft in each operation mode are obtained from the engine emission database of ICAO.

The ideal LTO (landing and takeoff) cycle is divided into four stages in the international standard atmosphere, and each has different engine power settings. This paper only studies the approach stage and takes only the approach process data of various types of engines.

There is a different type of engine matching among the aircraft teams of civil aviation airlines [16]. The matching data between the aircraft model and the engine are from the annual report of the airworthiness approval department of CAAC aircraft, and the time is up to October 1, 2020. The details are shown in Table 3 .

The civil aviation engine emission database is shown in Table 4.

MES (Manufacturing Execution System) system is a realtime information acquisition system, which develops rapidly in recent years. The total fuel consumption of an aeroengine equipped with an MES system is calculated by collecting relevant engine operation information in real time as follows: 


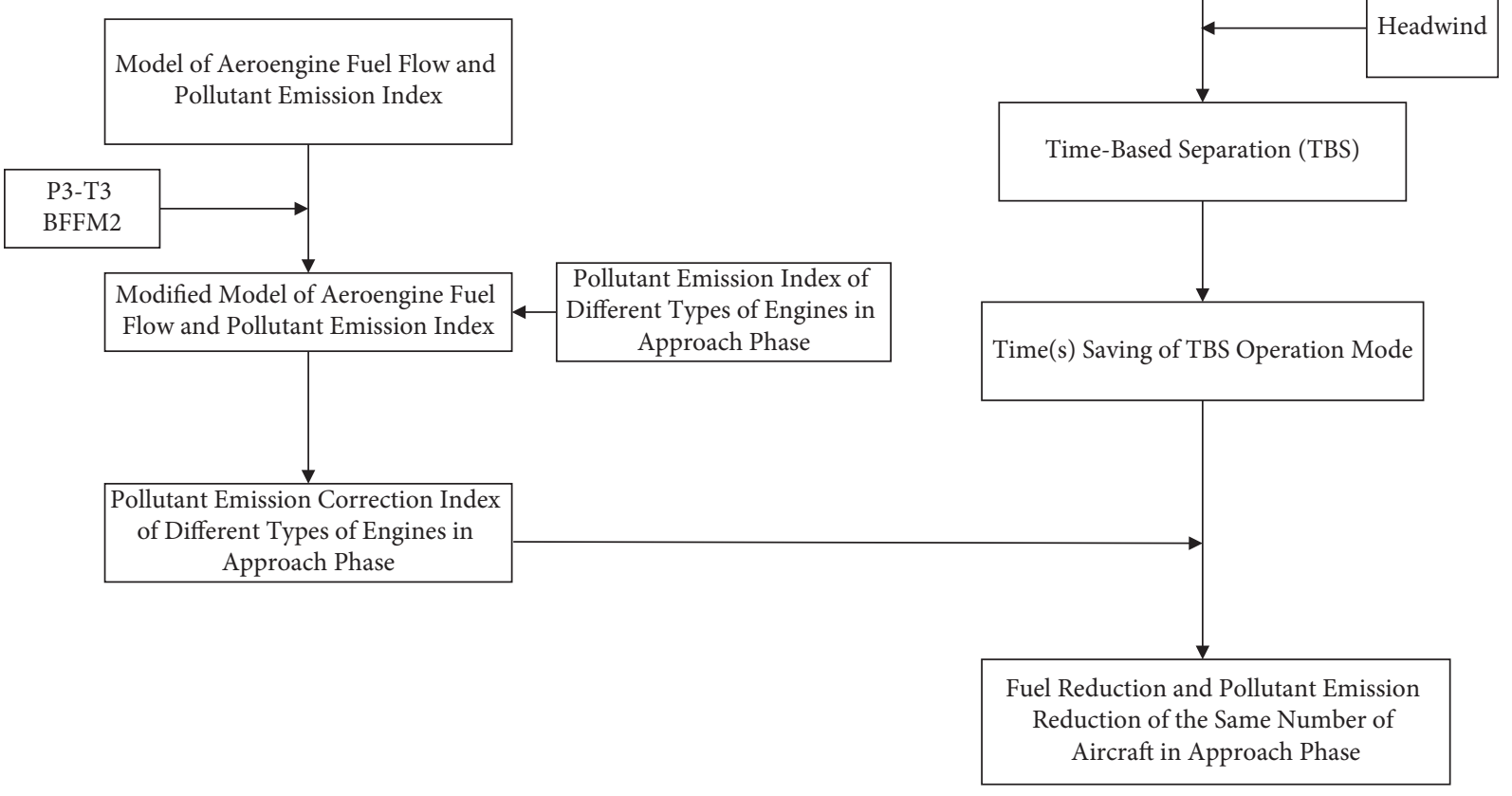

Figure 1: The structure flow chart of the article.

TABLE 1: Average approach speeds of various types of aircraft.

\begin{tabular}{lcc}
\hline Aircraft type & \multicolumn{2}{c}{ Average approach speeds } \\
\hline Light & 107 & Km/h \\
Middle & 145 & 200 \\
Heavy & 161 & 270 \\
A380 & 189 & 300 \\
\hline
\end{tabular}

TABLE 2: Engine thrust setting and working time under different working conditions of standard LTO cycle specified by ICAO [15].

\begin{tabular}{lcc}
\hline Flight phase & Thrust setting (\%) & Working time (min) \\
\hline Take off & 100 & 0.7 \\
Climb & 85 & 2.2 \\
Approach & 30 & 4 \\
Gliding & 7 & 26 \\
\hline
\end{tabular}

TABle 3: Comparison table of civil aviation aircraft/engine.

\begin{tabular}{lccccc}
\hline Aircraft Types & Engine Types & Aircraft Types & Engine Types & Aircraft Types & Engine Types \\
\hline A319 & CFM56-5A5 & B763/76L & PW4060 & B747 & RB211-524D4 \\
A320/32D & CFM56-5A1 & B767 & PW4056 & B757 & PW2040 \\
A321 & CFM56-5B3/P & B772/B773/B777 & Trent 892 & 7M8 & LEAP-1B28 \\
A330/33W/A332/A333 & CF6-80E1A2 & B77W/B777-300ER & GE90-115B & MD80 & JT8D-209 \\
A340/A343 & CFM56-5C4 & B787/78A/B788/B789 & Trent 1000-C & CRJ & CF34-8C5 \\
A346 & TRENT 556-61 & ERJ & AE3007A & B712 & BR700-715A1-30 \\
A350 & XWB-97 & JET & IAE V2500-A1 & A310 & CF6-80C2A2 \\
A380/A388 & GP7270 & B748 & GEnx-2B67B & B735 & CFM56-3-B1 \\
B737 & CFM56-3C-1 & B752/T204 & RB211-535E4 & MD82 & JT8D-217 \\
B738/B739 & CFM56-7B26 & B764 & CF6-80C2B8F & E190 & CF34-10E6 \\
B744 & CF6-80C2B1F & E70 & CF34-8E & & \\
\hline
\end{tabular}


TABle 4: Pollutant emission index of different engine models during approach.

\begin{tabular}{|c|c|c|c|c|c|c|c|}
\hline Engine model & Fuel flow $(\mathrm{kg} / \mathrm{s})$ & UHC $(\mathrm{g} / \mathrm{kg})$ & $\mathrm{CO}(\mathrm{g} / \mathrm{kg})$ & Engine model & Fuel flow $(\mathrm{kg} / \mathrm{s})$ & UHC $(\mathrm{g} / \mathrm{kg})$ & $\mathrm{CO}(\mathrm{g} / \mathrm{kg})$ \\
\hline AE3007Al & 0.113 & 0.655 & 3.91 & V2530-A5 & 0.377 & 0.056 & 1.81 \\
\hline CF6-80C2A5 & 0.687 & 0.2 & 1.93 & V2533-A5 & 0.3901 & 0.052 & 1.654 \\
\hline CF6-80C2-B6 & 0.686 & 0.2 & 1.93 & V2525-D5 & 0.319 & 0.061 & 2.44 \\
\hline CF34-3B & 0.116 & 0.13 & 1.88 & Trent772B/C-60 & 0.85 & 0.01 & 0.89 \\
\hline CF34-8Cl & 0.1681 & 0.06 & 2.91 & Trent556-61 & 0.62 & 0 & 0.46 \\
\hline CFM56-5B3/P & 0.366 & 0.5 & 1.7 & PW2037 & 0.458 & 0.11 & 1.95 \\
\hline CFM56-5B4/P & 0.312 & 0.5 & 2.3 & PW306B & 0.0974 & 0 & 7.11 \\
\hline CFM56-5B5/P & 0.26 & 0.7 & 3.4 & PW4052 & 0.6 & 0.17 & 2.15 \\
\hline CFM56-5B/6P & 0.275 & 0.6 & 2.9 & PW4056 & 0.658 & 0.13 & 2 \\
\hline CFM56-5B7/P & 0.312 & 0.5 & 2.3 & PW4060 & 0.703 & 0.14 & 1.78 \\
\hline CFM56-5C4 & 0.386 & 0.065 & 1.4 & PW4062 & 0.725 & 0.14 & 1.69 \\
\hline CFM56-3Bl & 0.338 & 0.1 & 1.6 & PW4062A & 0.708 & 0.15 & 1.68 \\
\hline CFM56-3B2 & 0.314 & 0.073 & 3.4 & PW4077D & 0.835 & 0.07 & 0.6 \\
\hline CFM56-3Cl & 0.336 & 0.07 & 3.1 & PW4158 & 0.682 & 0.14 & 1.88 \\
\hline CFM56-7B22 & 0.298 & 0.1 & 2.5 & JT9D-7R4E & 0.6529 & 0.13 & 1.23 \\
\hline CFM56-7B20 & 0.274 & 0.1 & 3.2 & JT8D-217A & 0.3833 & 0 & 3.54 \\
\hline CFM56-7B24 & 0.316 & 0.1 & 2.2 & JT8D-217C & 0.363 & 0 & 3.79 \\
\hline CFM56-7B26 & 0.338 & 0.1 & 1.6 & GE90-76B & 0.781 & 0.07 & 1.32 \\
\hline CFM56-7B27 & 0.349 & 0.1 & 1.4 & GE90-90B & 0.899 & 0.07 & 0.98 \\
\hline V2522-A5 & 0.311 & 0.062 & 2.6 & LF507-1H & 0.1083 & 0.12 & 4.43 \\
\hline V2524-A5 & 0.328 & 0.061 & 2.37 & RB211-535E4-37 & 0.52 & 0.04 & 1.14 \\
\hline V2527E-A5 & 0.319 & 0.061 & 2.44 & RB211-524H-36 & 0.77 & 0 & 1.05 \\
\hline
\end{tabular}

$$
\mathrm{NFC}_{m}=\sum_{a} N_{a} \times \mathrm{NFF}_{a, m} \times T_{a, m}
$$

Among them, $N_{a}$ is the engine number of $a$ aircraft, $T_{a, m}$ is the approach and landing time of $a$ aircraft under standard conditions, and $\mathrm{NFF}_{a, m}$ is the single-engine fuel flow $(\mathrm{kg} / \mathrm{s})$ of $a$ aircraft under standard conditions.

The pollutant $j$ emission $\mathrm{NE}_{a, j, m}$ is calculated as follows:

$$
\mathrm{NE}_{a, j, m}=\sum_{a} N_{a} \times \mathrm{NFF}_{a, m} \times T_{a, m} \times \mathrm{NEI}_{a, m, j}
$$

The $\mathrm{NEI}_{a, m, j}$ is the pollutant $j$ emission index of an aircraft engine under standard conditions.

2.1.3. Correction of Aeroengine Fuel Flow and Pollutant Emission Index. The relative method uses the known engine emission indexes under various thrust conditions on the ground, then modifies the model according to the ratio of some key thermal parameters of aircraft under high altitude and ground conditions and finally calculates the operational engine emission indexes under various actual conditions. The engine pollutant emission data by the ICAO at $30 \%$ thrust level on the ground are shown in Table 3. The engine emission index of the actual environment is obtained by correcting the P3-T3 emission index and Boeing Fuel Flow Method 2 (BFFM2) [17]. The study uses BFFM2 to calculate the fuel flow and pollutant emission index. Under international standard atmospheric conditions, the fuel flow and pollutant emission indices are based on the ideal LTO cycle.

The BFFM2 model needs to correct the approach stage's fuel flow, and the approach stage's correction coefficient is 1.020. Therefore, the revised fuel flow is linear with the thrust level of the engine [18].
Under the international standard atmosphere (ISA) condition, the modified single-engine fuel flow model converts sea-level fuel flow into actual fuel flow. The singleengine fuel flow correction expression is stated as follows:

$$
\begin{aligned}
\mathrm{NFF}_{a 0} & =\frac{\mathrm{NFF}_{a}}{\delta} \times \theta^{3.80} \times e^{V^{2}} \\
\delta & =\frac{p}{101.325} \\
\theta & =\frac{T+273.15}{288.15}
\end{aligned}
$$

Among them, $\mathrm{NFF}_{a 0}$ is the single-engine fuel flow correction value $(\mathrm{kg} / \mathrm{s})$. The $\mathrm{NFF}_{a}$ is the fuel flow under sealevel conditions $(\mathrm{kg} / \mathrm{s}) . \delta$ is the ratio between airport ground ambient pressure $(\mathrm{P})$ and sea-level pressure $(101.325 \mathrm{kPa})$ under International standard atmosphere (ISA). $\theta$ is the ratio between airport ground ambient temperature $(T+273.15)$ and the ISA sea-level temperature $(288.15 \mathrm{~K}) . V$ Is the aircraft speed of approach (Mach).

2.1.4. P3-T3 Emission Index Correction Model of Various Pollutants. NOx emission index estimation model: The $\mathrm{NOx}$ is mainly generated in the high-temperature flame zone of the primary combustion zone. It is related to the central combustion zone temperature, combustion chamber inlet temperature, combustion chamber pressure, fuel atomization quality, and residence time. The gas residence time in the high-temperature zone has an important influence on the generation of NOx [19]. The modified model $E_{1}$ of NOx emission index is as follows: 


$$
\begin{aligned}
E_{1} & =E_{1, r}\left(\frac{P_{3}}{P_{3, r}}\right)^{0.5}\left(\frac{T_{3, r}}{T_{3}}\right)^{0.5}\left(\frac{T_{1, r}}{T_{1}}\right)^{0.5} * \exp \left[\frac{38000}{\left(1 / T_{2, r}-1 / T_{2}\right)}\right], \\
T_{1} & =0.5\left(T_{2}+T_{3}\right), \\
T_{2} & =\operatorname{RT}(\varphi)+(1-R) T_{4}, \\
E_{1, r} & =d\left(\frac{P_{3}}{T_{3}}\right)^{0.5} T_{1}^{-1.5} e^{-38000 / T_{2}}=\mathrm{d} \lambda,
\end{aligned}
$$

where $\mathrm{d} \lambda$ is obtained by ICAO data fitting, as shown in Figure 2. $T_{1}$ and $T_{2}$ are the primary combustion zone and flame temperature. The variable with subscript " $r$ " is the reference value of the corresponding variable underground reference state. $T_{4}$ is the temperature when the primary combustion zone equivalence ratio is exactly $1 . T(\varphi)$ Is the primary combustion zone temperature when the equivalence ratio is $\varphi . R$ is the weight factor, generally 0.25 .

$\mathrm{CO}$ and UHC emission index estimation model. Both $\mathrm{CO}$ and UHC are generated by incomplete combustion of fuel. The $\mathrm{CO}$ is the hydrocarbon oxidation intermediate product. The UHC is mainly composed of incompletely burned fuel particles, fuel vapor and small molecular fuel cracked during combustion [20]. The calculation model of $\mathrm{CO}$ and $\mathrm{UHC}$ emission index (E2, E3) is as follows:

$$
\begin{aligned}
\frac{E_{2}}{E_{3}} & =\left(\frac{E_{2, r}}{E_{3, r}}\right)\left(\frac{T_{3}}{T_{3, r}} \frac{P_{3, r}}{P_{3}}\right)^{0.4}, \\
\frac{E_{2, r}}{E_{3, r}} & =a \theta^{2}+b \theta+c, \\
\theta & =\frac{\omega_{\text {Air }}}{P_{3}^{1.8} e^{T_{3} / 300}},
\end{aligned}
$$

where $\omega_{\text {Air }}$ is the airflow; $a, b$, and $c$ are the emission parameter obtained by ICAO data fitting.

The $\mathrm{CO}$ and $\mathrm{UHC}$ variation trend with $\theta$ at a ground standstill is shown in Figures 3 and 4, respectively.

According to the reference emission data of the engine ground test published by ICAO, with the help of the engine performance model, we obtain the relevant thermodynamic parameters under actual operational conditions and the estimation model of the above three engine pollutant emission indexes. The model can calculate the engine pollutant emission index under the actual operational conditions and then estimate the engine total pollutant emission under the actual flight conditions [21].

The pollutant emission index of taxing aircraft engines is also related to airport ambient pressure, ambient temperature, saturated vapor pressure, and atmospheric relative humidity. Therefore, the correction results of the pollutant emission index calculated by formulas (4) and (5) need to be further corrected as follows [22]:

$$
\begin{aligned}
\mathrm{NEI}_{a, j, 0,0} & =\left\{\begin{array}{l}
\mathrm{NEI}_{a, j, 0} \times\left(\frac{\theta^{3.30}}{\delta^{1.02}}\right)^{x}, j=\mathrm{UHC}, \mathrm{CO}, \\
\mathrm{NEI}_{a, j, 0} \times\left(\frac{\delta^{1.02}}{\theta^{3.30}}\right)^{y} \times e^{H}, \quad j=\mathrm{NO}_{x},
\end{array}\right. \\
H & =-19.0 \times\left(\frac{0.62197058 \times \phi \times P_{V}}{P-\phi P_{V}}-0.0063\right) .
\end{aligned}
$$

The humidity correction factor $P_{V}$ is derived from the Magnus Hedden equation:

$$
P_{V}=6.107 \times 10^{(q T / b+T)},
$$

where $\mathrm{NEI}_{a, j, 0,0}$ is the final modified value of $\mathrm{NEI}_{a, j, 0}$. Indices $x$ and $y$ are the corresponding engine values in the P3-T3 method. The default value is $x=1, y=0.5$. In this article, $x$ and $y$ are set as the default values.

In Engineering Data Management System (EDMS) version 5.0.1, the BFFM2 method uses the Goff-Gratch equation to calculate saturated vapor pressure; the EDMS version 5.0.2 is modified by the Magnus Hedden equation to calculate saturated vapor pressure [23]. The EDMS version 5.0.2 also changes the exponential equation of humidity correction coefficient to equation (7), where $P_{V}$ is the saturation pressure of water vapor $(\mathrm{kPa}), T$ is the ambient temperature $\left({ }^{\circ} \mathrm{C}\right), a=7.5, b=237.3, \phi$ is the relative humidity of the atmosphere, $p$ is the total ambient pressure $(\mathrm{kPa})$, and $H$ is the humidity correction factor.

\subsection{Time-Based Separations}

2.2.1. Aircraft Classification and Wake Separation in China. China's current RECAT-CN (Re-categorization-CHINA, RECAT-CN) experimental standard has appropriately reduced the CAAC's (China Administrative Association of Cat) radar wake separation standard. According to the maximum takeoff weight and wingspan length, the RECAT$\mathrm{CN}$ classification standard is shown in Table 5. Under the MTOW (maximum take-off weight) standard of CAAC, the B767 (Boeing-767) belongs to heavy-duty aircraft, and the wake separation standard between B767 and ARJ21 (Advanced Regional Jet for twenty-first century) is $9.3 \mathrm{~km}$. Under the RECAT-CN standard, B767 belongs to type C, similar to the wake separation standard of ARJ21. The classification of domestic operational aircraft based on the RECAT-CN standard is shown in Table 6 [24].

At present, the domestic wake separation standard based on RECAT-CN is shown in Table 7 [25].

The TBS separation mode recovers the loss of landing rate caused by DBS separation mode under strong headwind, improves landing rate, and reduces approach landing time. 


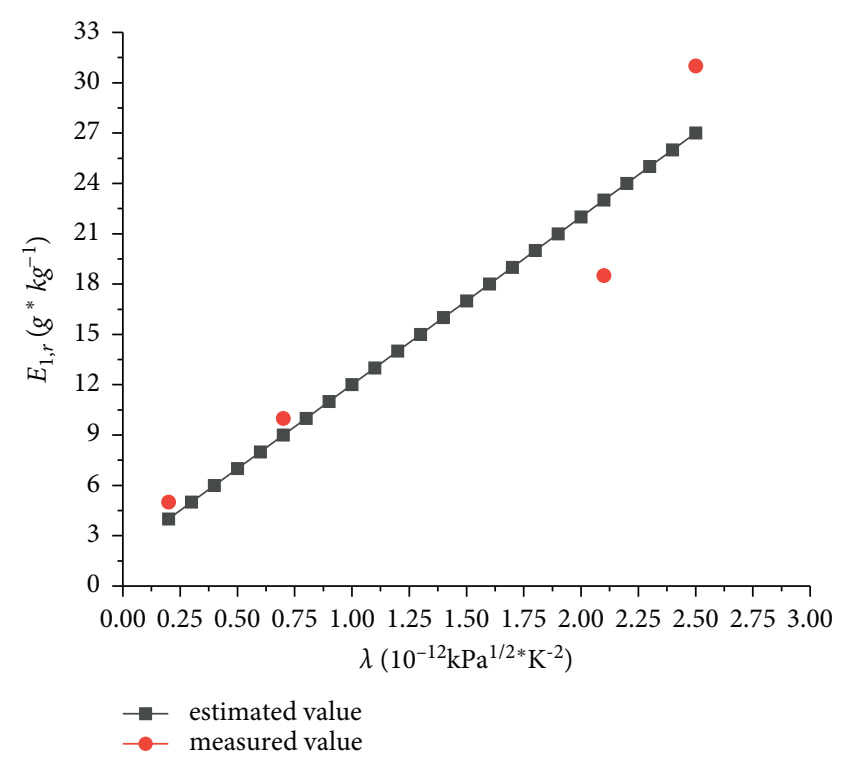

Figure 2: NOx reference emission index. $E_{1, r}$ curve with $\lambda$, red dot as measurement value, black box as the estimated value.

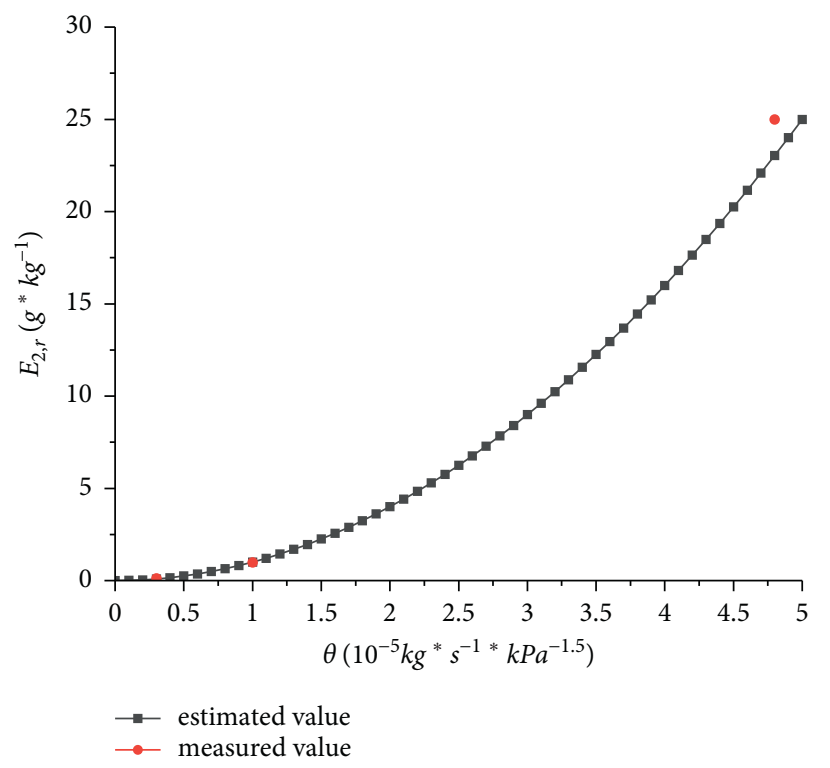

Figure 3: CO reference emission index. $E_{2, r}$ curve with $\theta$, red dot as measurement value, black box as the estimated value.

Some studies show that the headwind has little effect on landing efficiency when the headwind is below $15 \mathrm{kt}$. If the headwind is above $15 \mathrm{kt}$, it is recommended that the TBS separation mode replaces the DBS separation mode. The TBS is equivalent to the time required to fly the actual distance separation without wind [26]. The TBS separation mode is derived from the DBS separation mode, and the equivalent time separation standard is given by the following formula (8).

Suppose $D$ is a given distance separation, and GS is the aircraft ground speed, and $T$ is the time required to fly this distance.

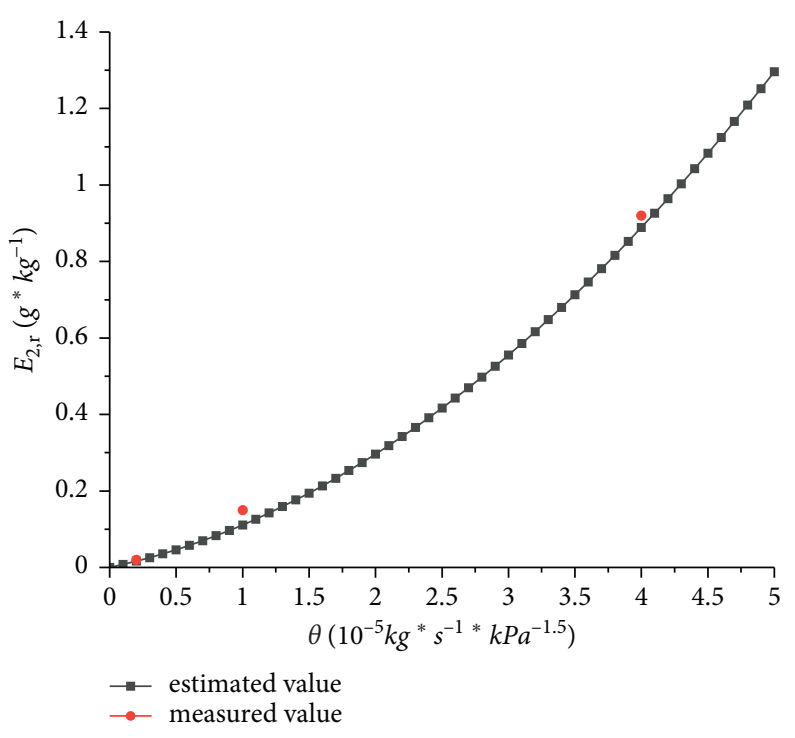

FIgURE 4: UHC reference emission index. $E_{3, r}$ curve with $\theta$, red dot as measurement value, black box as the estimated value.

TABle 5: RECAT-CN classification standard.

\begin{tabular}{lcc}
\hline RECAT-CN category & $($ MTOW $)(t)$ & Wing span a/m \\
\hline J & MTOW $\geq 136$ & $a \geq 75$ \\
B & MTOW $\geq 136$ & $54<a \leq 75$ \\
C & MTOW $\geq 136$ & $a \leq 54$ \\
M & $7<$ MTOW $\leq 136$ & \\
L & MTOW $\leq 7$ & \\
\hline
\end{tabular}

TABLE 6: Classification of domestic operational aircraft based on RECAT-CN standard.

\begin{tabular}{lc}
\hline $\begin{array}{l}\text { RECAT-CN } \\
\text { category }\end{array}$ & AircraftModel \\
\hline $\mathrm{J}$ & A380, AN225 \\
$\mathrm{B}$ & A330, B778, B779, B74 \\
$\mathrm{C}$ & A310, MD11, DC85, B703, B767 \\
$\mathrm{M}$ & ARJ21, C919, A320, B757-200, B757-300, B737, \\
$\mathrm{L}$ & CRJ, SSJ100, ER \\
\hline
\end{tabular}

$$
D=\mathrm{GS} \times T
$$

Under headwind conditions:

$$
\mathrm{GS}=\mathrm{IAS}-\mathrm{WS} \cos \alpha,
$$

where IAS (Indicated Airspeed) represents the aircraft's indicated airspeed, WS (Windspeed) is the wind speed (positive against the wind). $\alpha$ is the angle between wind direction and aircraft course. The time separation at different headwind speeds is as follows:

$$
T=\frac{D}{\text { IAS-WS } \cos \alpha} \text {. }
$$

During the final approach, the airspeed is set at $160 \mathrm{kt}$; the headwind speed of coastal airports often occurs between 
TABLE 7: Wake separation based on RECAT-CN (nautical miles).

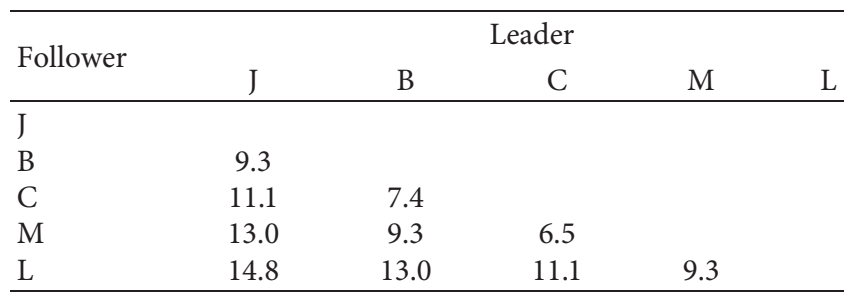

$15 \mathrm{kt}$ and $25 \mathrm{kt}$ in China [27]. Under low headwind conditions, the airport typical landing capacity is about 43 aircraft per hour. Increasing the $20 \mathrm{kt}$ headwind speed will reduce by 4 landing aircraft per hour. Under the stable average airspeed of $160 \mathrm{kt}$, the influence of the headwind on the final approach is shown in Table 8.

Table 9 shows the reduction of landing efficiency using DBS mode landing under $15 \mathrm{kt}, 25 \mathrm{kt}$, and $35 \mathrm{kt}$ headwind conditions compared to the reference landing rate at $160 \mathrm{kt}$ without headwind conditions.

It is found that the aircraft needs more time to fly at a given distance under strong headwind conditions so that airport will lose the stable runway capacity.

Under different headwind speed conditions, the same time separation will reduce the longitudinal distance separation. The details are shown in Figure 5, which help to maintain a constant runway capacity.

The wake distance stipulated by ICAO establishes the time separation standard. The detailed data are shown in Table 10. The minimum separation is 60 seconds to provide sufficient runway occupation time for aircraft ahead [28]. The detailed time separation standards of coastal airports in China are listed in Table 11.

Considering the constant distance separation, when the IAS is $136 \mathrm{kt}$, the time required to flight given distances is evaluated under constant headwind conditions of $0 \mathrm{kt}, 15 \mathrm{kt}$, and $25 \mathrm{kt}$. The results are shown in Table 12. When the headwind is stronger, the flight time required for a given distance is longer, and the flight arrival rate will be reduced [30]. Figure 6 shows the change of runway theoretical capacity (TC) with the change of ground speed at constant distance separations of $3,4,5$, and 6 nautical miles, where TC represents the maximum number of aircraft continuity landing per hour without other interference [31].

\subsubsection{Runway Arrival Capacity Model Based on Time Sep-} aration Standard. Under TBS mode, the separation of two aircraft is measured by time [32]. Therefore, no matter how the ground speed of the aircraft changes, the relative speed of the two aircraft will not affect the time separation between the two aircrafts [33]. The runway arrival capacity model is established under TBS mode as follows:
TABLE 8: Effect of headwind on a distance separation.

\begin{tabular}{lcccc}
\hline Mean Headwind (kt) & $\begin{array}{c}\text { Mean Ground Speed } \\
(\mathrm{kt})\end{array}$ & $\begin{array}{c}90 \mathrm{~s} \\
(\mathrm{Nm})\end{array}$ & $\begin{array}{c}113 \mathrm{~s} \\
(\mathrm{Nm})\end{array}$ & $\begin{array}{c}135 \mathrm{~s} \\
(\mathrm{Nm})\end{array}$ \\
\hline 5 & 160 & 4.0 & 5.0 & 6.0 \\
15 & 150 & 3.8 & 4.7 & 5.6 \\
25 & 140 & 3.5 & 4.4 & 5.3 \\
\hline
\end{tabular}

TABLE 9: Effect of headwind on land efficiency.

\begin{tabular}{lc}
\hline Mean Headwind (kt) & Time Spacing Impact (\%) \\
\hline 15 & 6.7 \\
25 & 14.3 \\
35 & 23.1 \\
\hline
\end{tabular}

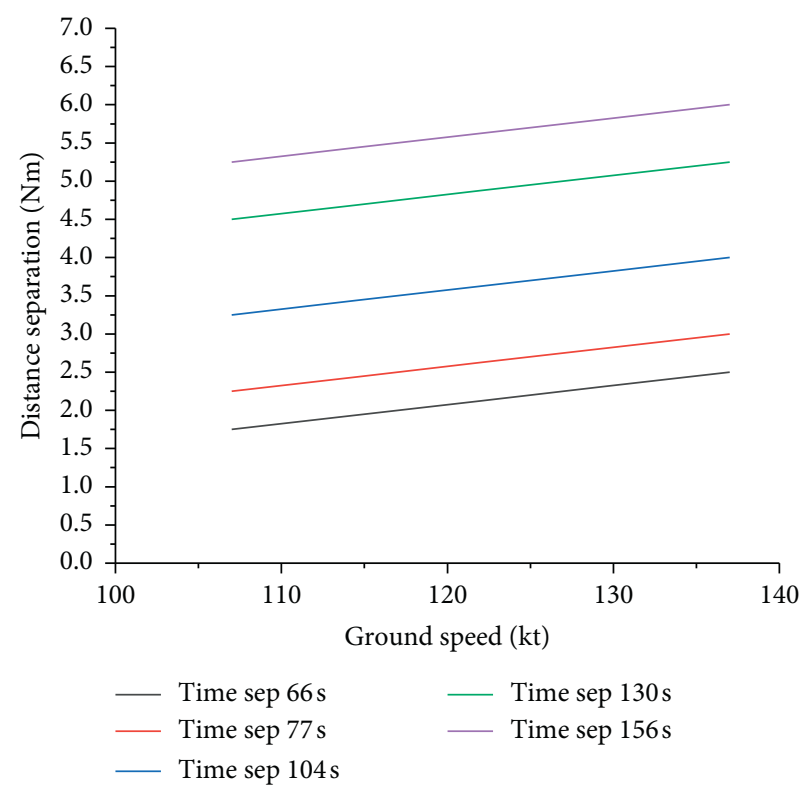

Figure 5: The same time separation reduces the longitudinal distance separation. With the decrease in ground speed, the distance separation becomes smaller at the same time separation.

$$
\begin{aligned}
& \lambda a=\frac{1}{\sum t_{i j}^{a} p_{i j}}, \\
& t_{i j}^{a}=\left\{\begin{array}{l}
\max \left(R_{a i}, t_{i j}\right), \quad v_{i} \leq v_{j}, \\
t_{i j}+\gamma\left(\frac{1}{v_{j}}-\frac{1}{v_{i}}\right), \quad v i>v j,
\end{array}\right. \\
& t_{i j}=\frac{\delta_{i j}}{v_{j}-w s \cos \alpha},
\end{aligned}
$$

where $\lambda_{a}$ is the airport landing capacity. $t_{i j}^{a}$ is the time separation of aircraft combination $i$ and $j$ (Leader is $i$ and Follower is $j$ ) passing through the runway entrance 
TABLE 10: Time separation standard [29].

\begin{tabular}{lcccc}
\hline \multirow{2}{*}{ Leader } & & \multicolumn{3}{c}{ Follower } \\
& A380 $(560 \mathrm{~T})(\mathrm{s})$ & Heavy (more than 136T) $(\mathrm{s})$ & Medium (7T to 136T) $(\mathrm{s})$ & Light $(7 \mathrm{~T}$ or less) $(\mathrm{s})$ \\
\hline A380 & 60 & 145 & 167 & 189 \\
Heavy & 60 & 98 & 60 & 145 \\
Medium & 60 & 60 & 60 & 122 \\
Light & 60 & 60 & 60 & 6 \\
\hline
\end{tabular}

TABLE 11: Time separation operation standard of coastal airports in China.

\begin{tabular}{|c|c|c|c|c|c|c|}
\hline \multirow[b]{2}{*}{ Leader } & \multicolumn{6}{|c|}{ Follower } \\
\hline & $\begin{array}{l}\text { A380 } \\
560 \mathrm{~T}\end{array}$ & $\begin{array}{c}\text { Heavy More than } \\
162 \mathrm{~T}\end{array}$ & $\begin{array}{l}\text { Upper Medium 104T to } \\
162 \mathrm{~T}\end{array}$ & $\begin{array}{c}\text { Lower Medium } 40 \mathrm{~T} \text { to } \\
104 \mathrm{~T}\end{array}$ & $\begin{array}{c}\text { Small } 17 \mathrm{~T} \text { to } \\
40 \mathrm{~T}\end{array}$ & $\begin{array}{c}\text { Light } 17 \mathrm{~T} \text { or } \\
\text { less }\end{array}$ \\
\hline A380 (JJ) & $90 \mathrm{~s}^{*}$ & $135 \mathrm{~s}$ & $158 \mathrm{~s}$ & $158 \mathrm{~s}$ & $158 \mathrm{~s}$ & $180 \mathrm{~s}$ \\
\hline Heavy $(\mathrm{HH})$ & $90 \mathrm{~s}^{*}$ & $90 \mathrm{~s}$ & $113 \mathrm{~s}$ & $113 \mathrm{~s}$ & $135 \mathrm{~s}$ & $158 \mathrm{~s}$ \\
\hline $\begin{array}{l}\text { Upper Medium } \\
\text { (UM) }\end{array}$ & $60 \mathrm{~s}^{*}$ & $60 \mathrm{~s}^{*}$ & $68 s$ & $90 \mathrm{~s}$ & $90 \mathrm{~s}$ & $135 \mathrm{~s}$ \\
\hline $\begin{array}{l}\text { Lower Medium } \\
\text { (LM) }\end{array}$ & $60 \mathrm{~s}^{*}$ & $60 \mathrm{~s}^{*}$ & $60 \mathrm{~s}^{*}$ & $60 \mathrm{~s}^{*}$ & $68 s$ & $113 \mathrm{~s}$ \\
\hline Small (SS) & $60 s^{*}$ & $60 \mathrm{~s}^{*}$ & $60 \mathrm{~s}^{*}$ & $60 \mathrm{~s}^{*}$ & $68 \mathrm{~s}$ & $90 \mathrm{~s}$ \\
\hline Light (LL) & $60 s^{*}$ & $60 \mathrm{~s}^{*}$ & $60 \mathrm{~s}^{*}$ & $60 \mathrm{~s}^{*}$ & $60 \mathrm{~s}^{*}$ & $60 \mathrm{~s}^{*}$ \\
\hline
\end{tabular}

${ }^{*} 90 \mathrm{~s}$ is the runway occupation time of special landing aircraft; $60 \mathrm{~s} *$ is the runway occupation time of landing aircraft.

TABLE 12: Effect of headwind on time separation [29].

\begin{tabular}{lcccc}
\hline Separation in NM & 3.0 & 4.0 & 5.0 & 6.0 \\
\hline $\begin{array}{l}\text { No wind } \\
\text { GS }=136 \mathrm{kts} \\
\text { Separation time (s) }\end{array}$ & 79 & 106 & 132 & 159 \\
\hline $\begin{array}{l}\text { Headwind 15 kt } \\
\text { GS }=121 \text { kts }\end{array}$ & 89 & 119 & 149 & 179 \\
Separation time (s) & & & & \\
\hline $\begin{array}{l}\text { Headwind 25 kt } \\
\text { GS }=111 \text { kts }\end{array}$ & 97 & 130 & 162 & 195 \\
Separation time (s) & & & & \\
\hline
\end{tabular}

successively. $p_{i j}$ is the proportion of front and rear aircraft of type $i$ and $j . v_{i}$ and $v_{j}$ are the average speed of final approach and landing of type $i$ an $j$ aircraft. $R_{a i}$ is the average time of $i$ aircraft runway occupation. $\delta_{i j}$ is the wake separation of different types of aircraft. $w s$ is the headwind speed, and $\alpha$ is the angle between the wind direction and the aircraft heading.

\section{Results and Discussion}

The direction and speed of wind have a significant impact on the aircraft approach process. The data of Heathrow Airport confirmed that the aircraft's ground speed decreases under the condition of increasing headwind. Under the same approach distance, the aircraft needs more time to complete the given separation distance than the condition of calm wind, resulting in the reduction of runway capacity.

In this paper, Shanghai Pudong Airport is selected as an example to analyze the landing data of one hour during peak hours. The Pudong Airport is a coastal airport, and windy weather is common. In this paper, using the landing data of Pudong Airport analyze the impact of headwind weather on

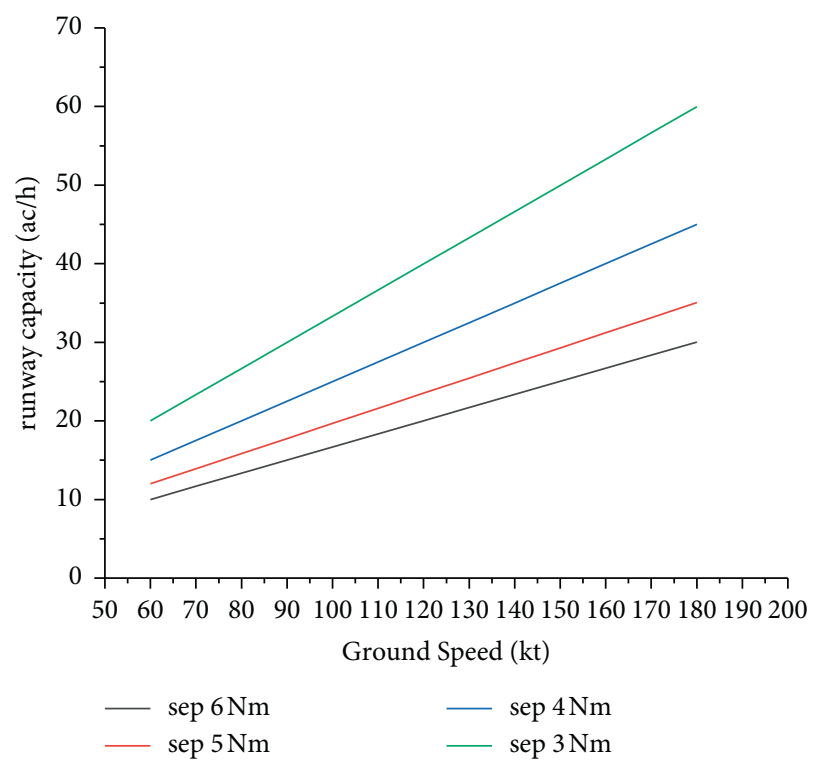

FIgURE 6: Variation of runway capacity with the ground speed at different distance separations. With the decrease in ground speed, the runway capacity decreases at the same distance separation.

the approach. The headwind speed data of Pudong Airport are shown in Table 13.

The broken line diagram of the runway time-varying headwind component is shown in Table 14. The direction of the Pudong Airport runway is $167^{\circ}$ and $347^{\circ}$, respectively. The velocity adopts the headwind component in the runway direction.

According to the average headwind speed from January 11 to February 13, the result shows a significant headwind speed on January 31 and February 7, and the average 
Table 13: Pudong Airport wind speed change during the period from January 11, 2019 to February 13, 2019.

\begin{tabular}{lccccccc}
\hline Date & Wind speed $(\mathrm{m} / \mathrm{s})$ & Date & Wind speed $(\mathrm{m} / \mathrm{s})$ & Date & Wind speed $(\mathrm{m} / \mathrm{s})$ & Date & Wind speed $(\mathrm{m} / \mathrm{s})$ \\
\hline 1.11 & 7.148505 & 1.19 & 8.105325 & 1.28 & 3.017721 & 2.06 & 7.204308 \\
1.12 & 2.817172 & 1.20 & 4.579507 & 1.29 & 9.856585 & 2.07 & 12.498351 \\
1.13 & 3.653269 & 1.21 & 0.628826 & 1.30 & 8.997928 & 2.08 & 3.753423 \\
1.14 & 5.702091 & 1.22 & 8.574796 & 1.31 & 12.925386 & 2.09 & 3.894943 \\
1.15 & 2.350936 & 1.23 & 1.389168 & 2.01 & 2.67304 & 2.10 & 4.837781 \\
1.16 & 8.953539 & 1.24 & 6.970074 & 2.02 & 8.220679 & 2.11 & 3.548583 \\
1.17 & 3.51852 & 1.25 & 5.238264 & 2.03 & 1.887818 & 1.17 \\
1.17 & 4.948712 & 1.26 & 4.255304 & 2.04 & 6.121343 & 2.12 & 3.885039 \\
1.18 & 7.148505 & 1.27 & 8.105325 & 2.05 & 1.733612 & 2.13 & 6.223767 \\
\hline
\end{tabular}

${ }^{*}$ In the date column, 1.11 means January $11,1.12$ means January 12 , and so on.

TABLE 14: Headwind component on runway.

\begin{tabular}{lccccccc}
\hline Date & Wind speed $(\mathrm{m} / \mathrm{s})$ & Date & Wind speed $(\mathrm{m} / \mathrm{s})$ & Date & Wind speed $(\mathrm{m} / \mathrm{s})$ & Date & Wind speed $(\mathrm{m} / \mathrm{s})$ \\
\hline 1.11 & 6.583132 & 1.19 & 7.236123 & 1.28 & 1.583214 & 2.06 & 4.238523 \\
1.12 & 1.968723 & 1.20 & 4.023426 & 1.29 & 8.112342 & 2.07 & 12.02152 \\
1.13 & 3.463286 & 1.21 & 0.223456 & 1.30 & 7.246653 & 2.08 & 2.954362 \\
1.14 & 4.259632 & 1.22 & 6.442562 & 1.31 & 11.98635 & 2.09 & 3.112596 \\
1.15 & 1.968572 & 1.23 & 0.983423 & 2.01 & 2.012631 & 2.10 & 2.042561 \\
1.16 & 8.265923 & 1.24 & 8.125328 & 2.02 & 7.013322 & 2.11 \\
1.17 & 3.027582 & 1.25 & 4.355212 & 2.03 & 1.003275 & 1.17 & 3.112853 \\
1.17 & 4.023584 & 1.26 & 4.005687 & 2.04 & 4.565246 & 2.12 & 1.562895 \\
1.18 & 6.836512 & 1.27 & 7.552163 & 2.05 & 0.925146 & 2.13 & 5.843215 \\
\hline
\end{tabular}

${ }^{*}$ In the date column, 1.11 means January 11, 1.12 means January 12, and so on.

headwind speed is $8.0 \mathrm{~m} / \mathrm{s}$. So the data of January 31 was used for analysis.

The histogram of wind speed and runway headwind component on January 31 is shown in Figure 7. The relatively stable headwind speed periods are $10: 30-11: 30,17: 00-18$ : $00,20: 00-21: 00$. The average wind speed is $10.3 \mathrm{~m} / \mathrm{s}, 8.1 \mathrm{~m} / \mathrm{s}$, $5.9 \mathrm{~m} / \mathrm{s}$, about $20.02 \mathrm{kt}, 15.75 \mathrm{kt}, 11.47 \mathrm{kt}$, respectively.

According to the experimental data of laser radar at Heathrow airport, the TBS time is reduced by about $65 \mathrm{~s}$ when the wind speed reaches $20 \mathrm{kt}$. The relationship between headwind speed and time-saved is shown in Figure 8.

Considering A320 and B737 series models, the radar separation between them is $6 \mathrm{~km}$, their approach speed is $136 \mathrm{kt}$, and the time separation is $86 \mathrm{~s}$ under the static wind. Under headwind, the airspeed is constant, and the ground speed is reduced. According to the experimental data of Heathrow airport, the average time separation calculated is the $40 \mathrm{~s}$. Under the strong headwind condition, the efficiency is increased by TBS mode. The minimum time separation is defined as the $60 \mathrm{~s}$ to provide enough runway occupancy time for the front aircraft.

The theoretical bearing capacity of the $35 \mathrm{~L}$ runway in those periods is calculated according to the measured landing aircraft data. Table 15 shows the runway capacity by using TBS mode in different periods. Table 16 contains the fuel consumption and pollutant emission reduction of landing aircraft in different periods.

The fuel consumption reduction in different periods is shown in Figure 9(a), and the pollutant emission reduction is shown in Figure 9(b).

From the above tables, compared with the DBS operation mode in three different periods, it can be concluded that

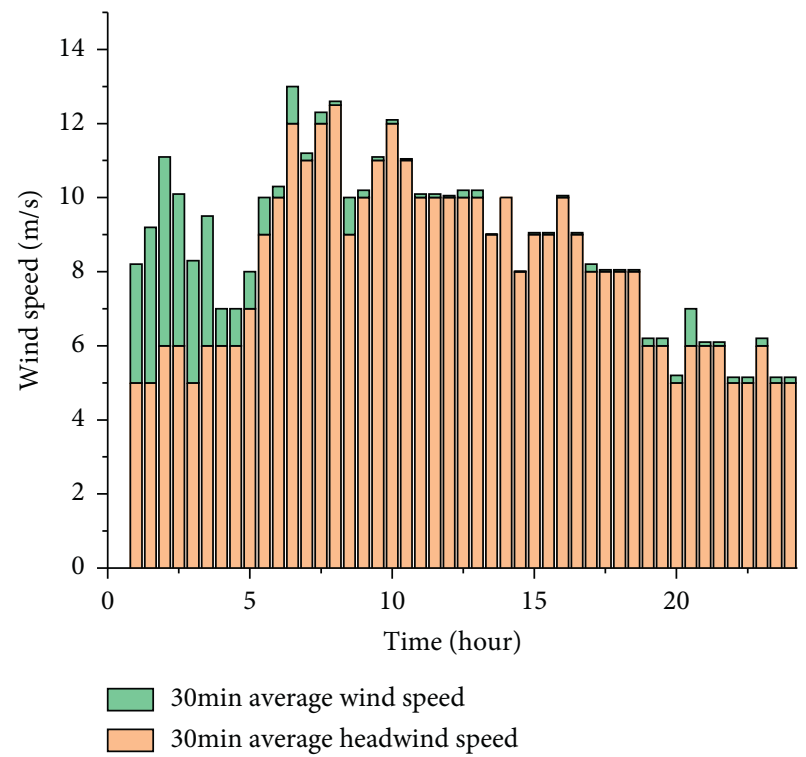

FIgURE 7: Within 30 min average wind speed of Shanghai Pudong Airport on January 31, 2019. The green column is the average wind speed within 30 minutes, and the red column is the wind speed against the runway.

the fuel saving of TBS operation mode is $858.64 \mathrm{~kg}$, $493.26 \mathrm{~kg}$, and $784.02 \mathrm{~kg}$; the emission reduction of $\mathrm{CO}$ is $2.58 \mathrm{~kg}, 1.48 \mathrm{~kg}$, and $2.35 \mathrm{~kg}$; the emission of $\mathrm{HC}$ is $0.53 \mathrm{~kg}$, $0.31 \mathrm{~kg}$, and $0.49 \mathrm{~kg}$; and the emission reduction of $\mathrm{NOx}$ is $7.91 \mathrm{~kg}, 4.54 \mathrm{~kg}$, and $7.22 \mathrm{k}$. The TBS operation mode can save many fuel costs for airlines and reduce many pollutant emissions for airports all over the country. 


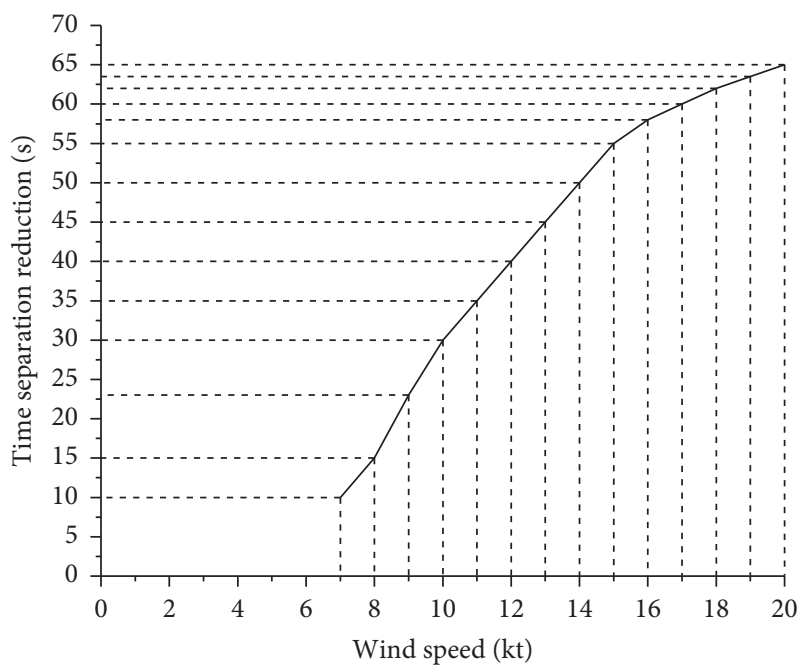

FIGURE 8: Relationship between headwind speed and time separation, the time separation model can save more time when the wind speed is stronger [34].

TABLE 15: Theoretical capacity of different periods.

\begin{tabular}{|c|c|c|c|}
\hline Time & $10: 30 \sim 11: 30$ & $17: 00 \sim 18: 00$ & $20: 00 \sim 21: 00$ \\
\hline Mean headwind speed & $10.3 \mathrm{~m} / \mathrm{s}(20.02 \mathrm{kt})$ & $8.1 \mathrm{~m} / \mathrm{s}(15.75 \mathrm{kt})$ & $5.9 \mathrm{~m} / \mathrm{s}(11.47 \mathrm{kt})$ \\
\hline Actual capacity of $35 \mathrm{~L}$ runway & 36 & 38 & 32 \\
\hline Time taken to land actual capacity based on time separation & $2427 \mathrm{~s}$ & $2913 \mathrm{~s}$ & $2505 \mathrm{~s}$ \\
\hline Time saving based on time separation & $1173 \mathrm{~s}$ & $687 \mathrm{~s}$ & $1095 \mathrm{~s}$ \\
\hline
\end{tabular}

TABLE 16: Emission reduction of various pollutants.

\begin{tabular}{|c|c|c|c|}
\hline Time $(\mathrm{kg})$ & $10: 30 \sim 11: 30$ & $17: 00 \sim 18: 00$ & $20: 00 \sim 21: 00$ \\
\hline Fuel saved based on time separation for the same sortie & 858.64 & 493.26 & 784.02 \\
\hline $\mathrm{CO}$ emission reduction & 2.58 & 1.48 & 2.35 \\
\hline HC emission reduction & 0.53 & 0.31 & 0.49 \\
\hline NOx emission reduction & 7.91 & 4.54 & 7.22 \\
\hline
\end{tabular}

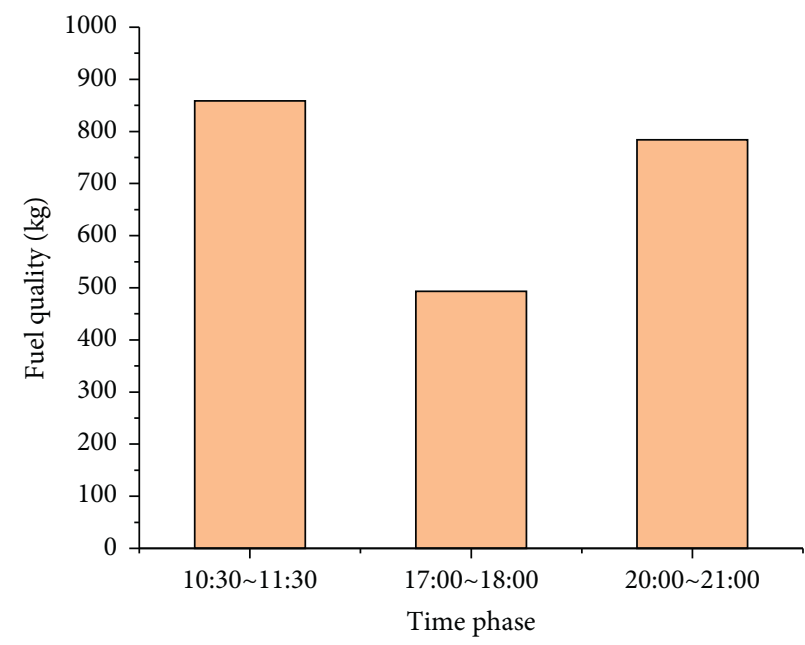

(a)

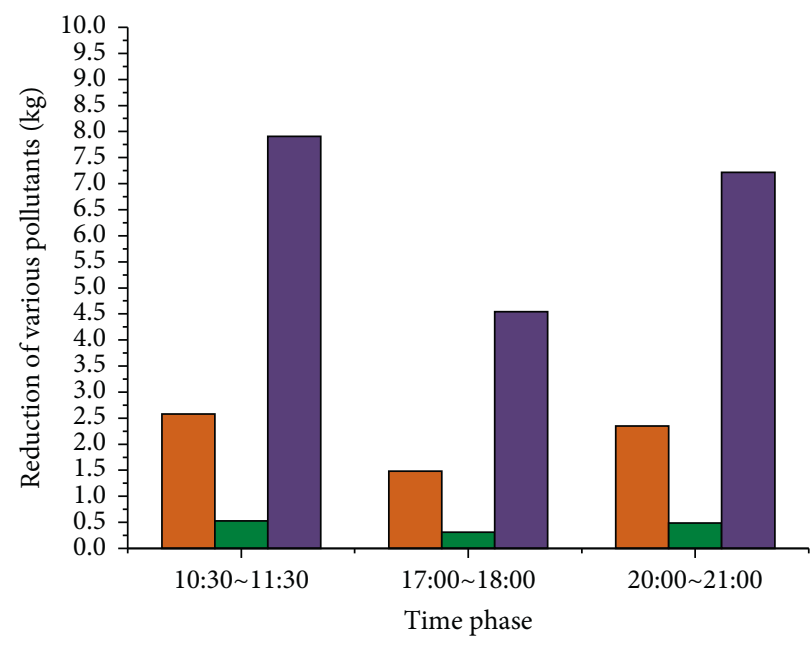

CO reduction $\mathrm{HC}$ reduction NOx reduction 
TABLE 17: Emission reduction percentage of fuel oil and various pollutants.

\begin{tabular}{lccc}
\hline Time & $10: 30 \sim 11: 30$ & $17: 00 \sim 18: 00$ & $20: 00 \sim 21: 00(\%)$ \\
\hline Fuel saving \% based on time separation for the same sortie & 32.52 & 19.12 & 30.41 \\
CO emission reduction\% & 28.93 & 17.9 & 29.29 \\
HC emission reduction\% & 31.02 & 19.36 & 33.78 \\
NOx emission reduction\% & 30.85 & 16.42 & 28.67 \\
\hline
\end{tabular}

Combined with Table 17, the statistical analysis in the three periods shows that the fuel saving is $32.52 \%, 19.12 \%$, and $30.41 \%$; the reduction of $\mathrm{CO}$ pollutant emissions is $28.93 \%, 17.9 \%$, and $29.29 \%$; the reduction of HC pollutant emission is $31.02 \%, 19.36 \%$, and $33.78 \%$; the reduction of NOx pollutant emission reduction is $30.85 \%, 16.42 \%$, and $28.67 \%$. For large coastal airports, we should pay more attention to energy conservation and emission reduction.[24]

\section{Conclusion}

(1) Compared with the DBS operation mode in the approach phase, the TBS operation mode will reduce fuel consumption by $10679.6 \mathrm{~kg}$, CO emission by $32.05 \mathrm{~kg}$, HC emission by $6.65 \mathrm{~kg}$, and NOx emission by $98.35 \mathrm{~kg}$.

(2) The TBS operation mode will make a significant contribution to the airport's energy conservation and emission reduction. They can reduce fuel consumption by at least $30 \%$ and pollutant emissions by $25 \%$.

(3) Considering the stronger headwind weather conditions, some coastal airports have greater fuel consumption and pollutant emissions, such as Qingdao Liu-ting Airport, Shanghai Pu-dong Airport, Xiamen Gao-Qi Airport, Guangzhou Bai-Yun Airport, and Dalian Zhou-Shuizi Airport. The airport or airline may consider implementing the TBS approach operation mode to reduce fuel and pollutant emissions.

(4) The further research content of this paper will take into account the influence of the changes of humidity and temperature in the actual atmospheric environment on the fuel consumption and pollutant emissions of the aeroengine. At present, this paper is based on the average approach speed of the same type of aircraft in the approach, and the next step study introduces the actual speed of aircraft in the process of approach into the model for calculation, which will improve the accuracy and objectivity of calculation. Finally, the aircraft's actual engine thrust and time are used to calculate to improve the accuracy of the results.

\section{Data Availability}

The data used in this paper are weather forecast data and airport monitoring data of Shanghai Pudong airport. The current research project provides these data. It is confidential and cannot be directly disclosed. It can be used for paper writing after processing. This paper uses the data measured by Morris et al. At Heathrow Airport about the relationship between headwind speed and time interval reduction.

\section{Conflicts of Interest}

No conflict of interest.

\section{Acknowledgments}

The authors acknowledge the financial support from the National Natural Science Foundation of China (Grant No. U1733203), Civil Aviation Safety Capacity Building Project (Grant No. (2020)170), Sichuan Science and technology project (Grant no. 2021YFS0319), and Central Government Guides Local Science and Technology Development Projects (Grant no. 2020ZYD094). The authors would like to thank the leaders and teachers of the Air Traffic Management School of Civil Aviation Flight College of China for their strong support and suggestions for this paper.

\section{References}

[1] D. Vechtel, "How future aircraft can benefit from a steerable main landing gear for crosswind operations," CEAS Aeronautical Journal, vol. 29, no. 328, pp. 1-13, 2019.

[2] G. Eason, B. Noble, and I. N. Sneddon, "On certain integrals of Lipschitz-Hankel type involving products of Bessel functions," Philosophical Transactions of the Royal Society of London, vol. A247, no. 1, pp. 529-551, 1955.

[3] J. Milan, "Toward time-based separation rules for landing aircraft," Transportation Research Record Journal of the Transportation Research Board, vol. 18, no. 2052, pp. 79-89, 2018.

[4] ICAO, "Redefinition of ICAO categories for wake turbulence," in Proceedings of the Tenth USA/Europe Twelth Air Navigation Conference, Montreal, Canada, November 2012.

[5] R. Fusaro, N. Viola, S. Cresto Aleina et al., "Innovative timebased separation procedures for civil RPAS integration," Aircraft Engineering \& Aerospace Technology, vol. 91, no. 1, 2018.

[6] C. Lunsford and D. Cynthia, "Enabling aircraft-based observation of winds in the US and Europe: progress and benefits," in Proceedings of the Digital Avionics Systems Conference (DASC), 2017 IEEE/AIAA 36thconf.IEEE, Petersburg, Florida, USA, September 2017.

[7] N. Khadka, S. Parajuli, A. Bhattarai et al., "A study of optimization of aircraft separation using the time-based separation at tribhuvan international airport," World Academics Journal of Engineering Sciences, vol. 7, 2020.

[8] Q. Xia, Study on the Impact of Aircraft Engine Emissions on Airport Atmospheric Environment, Nanjing University of Aeronautics and Astronautics, Nanjing, China, 2009. 
[9] Y. Huang, G. Zhou, and S. Wu, "A preliminary investigation on the inventory of NOx emitted from CAAC flights over China," Acta Scientiae Circumstantiae, vol. 2, 2018.

[10] Z. Wei and C. Wang, "Estimation method of pollutant emission in different flight stages," Journal of Transportation Engineering, vol. 10, no. 06, pp. 48-52, 2018.

[11] Y. Huang, D. Wu, and W. S. Wang, "Further study on NOx emission distribution of civil aviation aircraft over China," Journal of Beijing University of Aeronautics and Astronautics, vol. 12, no. 3, pp. 289-292, 2015.

[12] M. Sherry, A. Nemes, D. L. Jacono et al., "The interaction of helical tip and root vortices in a wind turbine wake," Physics of Fluids, vol. 24, no. 25, pp. 115-121, 2018.

[13] D. S. Hicok and B. E. Barmore, "Concept of operations for separation management arrivals and approach," Physics of Fluids, vol. 28, no. 7, pp. 78-841, 2016.

[14] L. J. Zhong, J. Y. Zheng, and G. Q. Lei, "Quantitative uncertainty analysis in air pollutant emission inventories: methodology and case study," Research of Environmental, vol. 24, no. 4, pp. 15-20, 2017.

[15] L. Chen, "Estimation and prediction of China's air transport emissions in LTO and cruise phases," Journal of Beijing Jiaotong University, vol. 12, no. 04, pp. 27-33, 2013.

[16] Q. H. Zuo, F. Yang, and W. J. Pan, "Review on the development of civil aircraft wake reclassification," Henan science and technology, vol. 39, no. 26, pp. 65-70, 2020.

[17] W. J. Pan, B. Ran, Z. Y. Wu, and X. P. Zhu, "Review of wake separation classification standards," Journal of Civil Aviation Flight College of China, vol. 30, no. 2, pp. 54-57, 2019.

[18] X. H. Tang, "Research on aircraft emission diffusion characteristics based on Gaussian puff model," Civil Aviation University of China, vol. 11, no. 2, pp. 22-28, 2020.

[19] Z. Y. Wu, "Research on aircraft wake vortex identification and prediction based on Doppler lidar," China Civil Aviation Flight College, vol. 18, no. 5, Article ID 200632, 2020.

[20] B. B. Li and R. T. Nie, "Analysis of the influence of time separation standard (TBS) on runway capacity," Aeronautical computing technology, vol. 45, no. 6, pp. 87-90, 2015.

[21] W. J. Pan, Y. A. Liang, H. J. Liang, W. X. Deng, and S. R. Zheng, "Safety analysis of single runway takeoff separation based on numerical calculation model," Aviation computing technology, vol. 49, no. 4, pp. 4-8, 2019.

[22] T. G. Zhang and X. Y. Hou, "Research on airworthiness certification method of aeroengine exhaust pollutants," Science and technology guide, vol. 30, no. 4, pp. 67-70, 2012.

[23] W. J. Pan, Z. W. Zhang, X. L. Zhang, and J. Y. Chen, "Improvement of Reich collision risk model under Re-cat wake separation standard," Aviation computing technology, vol. 49, no. 3, pp. 6-9, 2019.

[24] L. P. Jia and Q. Luo, "Experimental study on single head low pollution combustion chamber of aeroengine," Mechanical manufacturing and automation, vol. 49, no. 3, pp. 63-66, 2020.

[25] Z. Q. Zhao, J. Li, Y. Tian, and X. Y. Li, "Review of the research on the impact of aviation emissions on atmospheric environment," Environmental science and technology, vol. 41, no. 3, pp. 186-192, 2020.

[26] K. J. Yu, D. W. Yu, and M. Wang, "Research on Civil Aviation energy saving and emission reduction based on aircraft LTO cycle in airport area," Science and Technology, vol. 24, no. 12, pp. 27-32, 2015.

[27] H. L. Cao, J. Z. Gao, and D. M. Liang, "United States," OECD Science, Technology and Industry Outlook, vol. 37, no. 1, pp. 444-447, 2014.
[28] J. Z. Gao, "Research on emission and diffusion model of civil aviation engine in whole flight," Environmental science and technology, vol. 55, no. 3, pp. 29-33, 2019.

[29] X. Y. Hou and T. G. Zhang, "Calculation method for airworthiness certification of NOx emission from aero engine based on LTO cycle," Science and technology guide, vol. A30, no. 21 , pp. $61-65,2012$.

[30] C. Morris and J. Peters, "Validation of the time based separation concept at london heathrow Airport," in Proceedings of the 10th USA/Europe ATM R\&D Seminar, vol. 10, no. 13, pp. 32-38, Chicago, IL, USA, June 2013.

[31] J. Z. Sun, H. F. Zuo, P. P. Liu, and Y. Fu, "Estimation method of air engine pollutant emission," Journal of Transportation Engineering, vol. 12, no. 2, pp. 53-61, 2012.

[32] W. J. Pan, J. J. Zuo, Y. A. Liang, W. X. Deng, and H. J. Liang, "Dynamic response model and safety analysis of aircraft encountering wake," Journal of Ordnance Equipment Engineering, vol. 40, no. 6, pp. 211-214, 2019.

[33] Q. Xia, H. F. Zuo, and J. L. Yang, "Estimation of aircraft takeoff and landing (LTO) cycle emissions at CAAC airport," Journal of environmental science, vol. 1, no. 7, pp. 1469-1474, 2008.

[34] S. Q. Li and W. Hu, "Estimation method of air pollutant emission during air transportation," Statistical research, vol. 37, no. 1, pp. 63-64, 2014. 